\title{
Decomposition in Finite Series for Dedekind sum and Some Hardy sums by Trigonometric Functions
}

\section{Prof. Dr. K. Raja Rama Gandhi and Edigles Guedes}

${ }^{1}$ Resource person in Math for Oxford University Press, Professor in Math, BITS-Vizag.

${ }^{2}$ World order Number Theorist, Pernambuco, Brazil.

\section{INTRODUCTION}

The Dedekind sum is defined by

$$
s(h, k):=\sum_{\mu(\bmod k)}\left(\left(\frac{\mu}{k}\right)\right)\left(\left(\frac{h \mu}{k}\right)\right),
$$

being $h$ an integer and $k$ a positive integer and

$$
((x))=\left\{\begin{array}{c}
x-\lfloor x\rfloor-\frac{1}{2}, \text { if } x \notin \mathbb{Z}, \\
0, \text { otherwise, }
\end{array}\right.
$$

where $\lfloor x\rfloor$ is the floor function. then

By use of finite Fourier series, in [1] and [2], H. Rademacher proved that, for $\operatorname{gcd}(h, k)=1$,

$$
s(h, k)=\frac{1}{4 k} \sum_{r=1}^{k-1} \cot \left(\frac{\pi r}{k}\right) \cot \left(\frac{\pi h r}{k}\right)
$$

\section{Preliminares}

Lemma 1. If $c=\frac{a}{b}$ and $b \neq 0$, then

$$
a^{2} b+a b^{2} c^{2}=a^{3}+b^{3} c^{2} .
$$

Proof. Suppose that

$$
y=1+z y^{2},
$$

then, we have the parametrization

$$
z=\frac{t-1}{t^{2}}
$$

and

$$
y=t
$$

Let $t=\frac{a}{b}$ and $t^{2}=c^{2}$, such that $c=\frac{a}{b}$. Therefore,

$$
\begin{aligned}
& \frac{a}{b}=1+\frac{\frac{a}{b}-1}{c^{2}}\left(\frac{a}{b}\right)^{2} \\
& \frac{a}{b}=1+\frac{(a-b) a^{2}}{b^{3} c^{2}}
\end{aligned}
$$




$$
\begin{gathered}
\frac{a}{b}=\frac{b^{3} c^{2}+(a-b) a^{2}}{b^{3} c^{2}}, \\
a=\frac{b^{3} c^{2}+(a-b) a^{2}}{b^{2} c^{2}}, \\
a b^{2} c^{2}=b^{3} c^{2}+a^{3}-a^{2} b, \\
a^{2} b+a b^{2} c^{2}=a^{3}+b^{3} c^{2} .
\end{gathered}
$$

Lemma 2. For $x \in \mathbb{R}$, we have

$$
\begin{aligned}
& \cot \left(\frac{x}{2}\right)=\frac{1}{2}\left[\cot ^{2}\left(\frac{x}{2}\right)-1\right] \tan (x), \\
& \cot (x)=\frac{1}{2}\left[\cot ^{2}(x)-1\right] \tan (2 x),
\end{aligned}
$$

and

$$
\begin{aligned}
& \tan \left(\frac{x}{2}\right)=\frac{1}{2}\left[1-\tan ^{2}\left(\frac{x}{2}\right)\right] \tan (x), \\
& \tan (x)=\frac{1}{2}\left[1-\tan ^{2}(x)\right] \tan (2 x),
\end{aligned}
$$

Proof. We define that

$$
\tan (\mathrm{x}):=\frac{\sin (\mathrm{x})}{\cos (\mathrm{x})}
$$

and if we set

$$
t=\tan \left(\frac{x}{2}\right)
$$

then

$$
\begin{aligned}
& \sin (x)=\frac{2 t}{1+t^{2}}, \\
& \cos (x)=\frac{1-t^{2}}{1+t^{2}} \\
& \tan (x)=\frac{2 t}{1-t^{2}} .
\end{aligned}
$$

By Lemma 1, combined with (5), (6) and (7), we have

$$
\begin{gathered}
\sin (x)^{2} \cos (x)+\sin (x) \cos (x)^{2} \tan (x)^{2}=\left(\frac{2 t}{1+t^{2}}\right)^{3}+\left(\frac{1-t^{2}}{1+t^{2}}\right)^{3}\left(\frac{2 t}{1-t^{2}}\right)^{2} \\
\sin (x)^{2} \cos (x)+\sin (x)^{3}=8\left(\frac{t}{1+t^{2}}\right)^{3}+4 \frac{\left(1-t^{2}\right) t^{2}}{\left(1+t^{2}\right)^{3}} \\
\sin (x)^{2}[\cos (x)+\sin (x)]=\frac{4 t^{2}\left(-t^{2}+2 t+1\right)}{\left(1+t^{2}\right)^{3}} \\
\sin (x)^{2}[\cos (x)+\sin (x)]=-4 t\left(\frac{t}{1+t^{2}}\right)^{3}+8\left(\frac{t}{1+t^{2}}\right)^{3}+4 \frac{1}{1+t^{2}}\left(\frac{t}{1+t^{2}}\right)^{2}
\end{gathered}
$$




$$
\begin{gathered}
\sin (x)^{2}[\cos (x)+\sin (x)]=-4 t\left(\frac{\sin (x)}{2}\right)^{3}+8\left(\frac{\sin (x)}{2}\right)^{3}+4 \frac{1}{1+t^{2}}\left(\frac{\sin (x)}{2}\right)^{2} \\
\sin (x)^{2}[\cos (x)+\sin (x)]=-\frac{t}{2} \sin ^{3}(x)+\sin ^{3}(x)+\frac{1}{t}\left(\frac{t}{1+t^{2}}\right) \sin ^{2}(x) \\
\sin (x)^{2}[\cos (x)+\sin (x)]=-\frac{t}{2} \sin ^{3}(x)+\sin ^{3}(x)+\frac{1}{t}\left(\frac{\sin (x)}{2}\right) \sin ^{2}(x) \\
\sin (x)^{2}[\cos (x)+\sin (x)]=-\frac{t}{2} \sin ^{3}(x)+\sin ^{3}(x)+\frac{1}{2 t} \sin ^{3}(x)
\end{gathered}
$$

Eliminating $\sin ^{2}(x)$ in both members of (8) and manipulating algebraically, we encounter

$$
\begin{gathered}
2 \cos (x) t+2 \sin (x) t=-\sin (x) t^{2}+2 \sin (x) t+\sin (x) \\
-\sin (x) t^{2}-2 \cos (x) t+\sin (x)=0 \\
\sin (x) t^{2}+2 \cos (x) t-\sin (x)=0
\end{gathered}
$$

We can easily verify that the above equation has the following solutions

$$
t=-\cot \left(\frac{x}{2}\right), t=\tan \left(\frac{x}{2}\right)
$$

From (9) and (10), it follows that

$$
\cot \left(\frac{x}{2}\right)=\frac{\cot ^{2}\left(\frac{x}{2}\right)-1}{2 \cos (x)} \sin (x)=\frac{1}{2}\left[\cot ^{2}\left(\frac{x}{2}\right)-1\right] \tan (x)
$$

and

$$
\tan \left(\frac{x}{2}\right)=\frac{1-\tan ^{2}\left(\frac{x}{2}\right)}{2 \cos (x)} \sin (x)=\frac{1}{2}\left[1-\tan ^{2}\left(\frac{x}{2}\right)\right] \tan (x)
$$

To complete the proof let $x \rightarrow 2 x$ in the two previous equations.

\section{Dedekind sum}

Theorem 1. Let $\operatorname{gcd}(h, k)=1$, if $h$ is even and $k$ is odd, then

$$
\begin{aligned}
s(h, k)=\frac{1}{16 k} & \sum_{r=1}^{k-1}\left[\tan \left(\frac{2 \pi r}{k}\right) \tan \left(\frac{2 \pi h r}{k}\right) \cot ^{2}\left(\frac{\pi r}{k}\right) \cot ^{2}\left(\frac{\pi h r}{k}\right)\right. \\
& -\tan \left(\frac{2 \pi r}{k}\right) \tan \left(\frac{2 \pi h r}{k}\right) \cot ^{2}\left(\frac{\pi h r}{k}\right)-\tan \left(\frac{2 \pi r}{k}\right) \tan \left(\frac{2 \pi h r}{k}\right) \cot ^{2}\left(\frac{\pi r}{k}\right) \\
& \left.+\tan \left(\frac{2 \pi r}{k}\right) \tan \left(\frac{2 \pi h r}{k}\right)\right] .
\end{aligned}
$$

Proof. We substitute (3.a2) in (1), as follows

$$
s(h, k)=\frac{1}{16 k} \sum_{r=1}^{k-1}\left[\tan \left(\frac{2 \pi r}{k}\right) \cot ^{2}\left(\frac{\pi r}{k}\right)-\tan \left(\frac{2 \pi r}{k}\right)\right]\left[\tan \left(\frac{2 \pi h r}{k}\right) \cot ^{2}\left(\frac{\pi h r}{k}\right)-\tan \left(\frac{2 \pi h r}{k}\right)\right]
$$




$$
\begin{gathered}
=\frac{1}{16 k} \sum_{r=1}^{k-1}\left[\tan \left(\frac{2 \pi r}{k}\right) \tan \left(\frac{2 \pi h r}{k}\right) \cot ^{2}\left(\frac{\pi r}{k}\right) \cot ^{2}\left(\frac{\pi h r}{k}\right)-\tan \left(\frac{2 \pi r}{k}\right) \tan \left(\frac{2 \pi h r}{k}\right) \cot ^{2}\left(\frac{\pi h r}{k}\right)\right. \\
\left.-\tan \left(\frac{2 \pi r}{k}\right) \tan \left(\frac{2 \pi h r}{k}\right) \cot ^{2}\left(\frac{\pi r}{k}\right)+\tan \left(\frac{2 \pi r}{k}\right) \tan \left(\frac{2 \pi h r}{k}\right)\right] .
\end{gathered}
$$

\section{INSIGHTS WITHOUT PROOFS}

Let $\operatorname{gcd}(h, k)=1$, if $h$ is even and $k$ is odd, then

$$
\begin{aligned}
& s(h, k)=\frac{1}{16 k} \sum_{r=1}^{k-1}\left[\tan \left(\frac{\pi r}{k}\right) \cot \left(\frac{\pi h r}{k}\right) \cot ^{2}\left(\frac{\pi r}{k}\right)-\tan \left(\frac{2 \pi r}{k}\right) \cot \left(\frac{\pi h r}{k}\right)\right] \\
& s(h, k)=\frac{1}{16 k} \sum_{r=1}^{k-1}\left[\tan \left(\frac{\pi h r}{k}\right) \cot \left(\frac{\pi r}{k}\right) \cot ^{2}\left(\frac{\pi h r}{k}\right)-\tan \left(\frac{2 \pi h r}{k}\right) \cot \left(\frac{\pi r}{k}\right)\right] .
\end{aligned}
$$

\section{Hardy sums}

I not solve the Hardy sums, because I not have the Collected Papers of G. H. Hardy, Vol IV, in On Certain Series of Discontinuous Functions Connected with the Modular Functions. Then, I leave these problems for Sir.

\section{REFERENCES}

[1] Rademacher, Egy Reciprocitásképletröl a Modulfüggevenyek Elméletpeböl, Mat. Fiz. Lapok 40 (1933), pp. 24-34.

[2] Rademacher, H., Some remarks on certain generalized Dedekind sums, Acta Arith. 9 (1964), pp. 97-105. 